\title{
Illness Perceptions, Psychological Distress and Coping Strategies as Predictors of Quality of Life After Radiotherapy in Head and Neck Cancer Patients
}

\author{
Vassiliki Siafaka, MSc, PhD'; Maria Prodromou, MSc²; Kleoniki Katinioti, MD, PhD²; \\ Dionysios Tafiadis, MSc, PhD'; Nafsika Ziavra, MD, PhD'; Periklis Tsekeris, MD, PhD; \\ Thomas Hyphantis, MD, $\mathrm{PhD}^{3}$
}

1 Department of Speech and Language Therapy, School of Health Sciences, University of loannina, loannina, Greece 2 Department of Radiation Oncology, University Hospital of loannina, loannina, Greece ${ }^{3}$ Faculty of Medicine, School of Health Sciences, University of loannina, loannina, Greece

\section{ABSTRACT}

Objective: To investigate the predictive value of certain psychological factors on the quality of life (QoL) of patients with head and neck cancer (HNC) after radiotherapy.

Methods: Depressive and anxiety symptoms (HADS), illness perceptions (IPQ-R), coping strategies (Brief COPE) and QoL (EORTC-QLQ-C30 and EORTC-QLQ-H\&N35) were assessed in fifty patients before radiotherapy (T1) and 3 months afterwards (T2).

Results: Hierarchical regression analysis revealed that depression (HADS-D) at T1 was associated with impairment in Physical, Role, and Social Functioning $(P=0.025, P=0.019$ and $P=0.019$, respectively) at $T 2$. Additionally, Behavioral Disengagement (Brief COPE) at T1 was the coping strategy most closely associated with lower scores on Physical $(P=0.013)$, Emotional $(P=0.003)$, Cognitive $(P=0.042)$ and Social Functioning $(P=0.040)$. Also, regarding IPQ-R dimensions, stronger beliefs about Emotional Representations and weaker beliefs about Illness Coherence at T1 were associated with higher H\&N35 sum score at $T 2(P=0.024$ and $P=0.032$, respectively). Finally, stronger Perceived Control $(I P Q-R)$ at T1 was associated with higher Social Functioning at $T 2(P=0.011)$. Conclusion: Depressive symptoms are predictors of impaired QoL in patients with HNC after radiotherapy. Additionally, Behavioral Disengagement, as avoidance coping mechanism, and strong beliefs that HNC will affect their emotional well-being aggravate certain domains of the QoL, while belief that their illness is amenable to treatment is associated with better QoL following radiotherapy. Psychological intervention aimed at improving the QoL of patients with HNC undergoing radiotherapy should be based on identification of depression in these patients, and their coping strategies and illness perceptions.
\end{abstract}

\section{INTRODUCTION}

Head and neck cancer (HNC) is a heterogeneous group of malignancies that accounts for $5 \%$ of malignant diseases. It shows a higher prevalence in men, being the fourth most frequent cancer in the male population; its incidence increases with age ( $>60$ years) and is associated with abuse of tobacco and alcohol. Radiotherapy (RT) is the main form of treatment, either alone or combined with surgery and/or chemotherapy, and is reported to promote the quality of life (QoL) of patients by alleviating their symptoms. The adverse effects of HNC and its treatment are related to vital functions, and include difficulties in swallowing, breathing and speech, but are also associated with such emotional dimensions as negative self-image, psychological distress, and social isolation. Many patients experience increased levels of psychological distress as they face stressful medical interventions, repetitive and different types of treatments and side effects, and go through the different stages of the disease. As a result, they often reflect a type of "burnout", i.e., a psychological deterioration as a consequence of chronic stress [1]. These effects force individuals with $\mathrm{HNC}$ to adapt to their new situation at the social and existential level, searching for coping strategies to maximize the adaptation to the disease and a meaning in their disease experience [2].

Individuals process the situation and, finally, adapt to their illness in various different ways, irrespective of their clinical and socio-demographic char- acteristics. In addition, their coping strategies are determined by a variety of factors, such as personality, religiousness and received support. According to the Common Sense Model [3], in order to adapt to a new situation the individual engages in a dynamic process of reconstruction of ideas, feelings and behavior, which can influence various dimensions of their QoL [4].

Factors that are reported to influence the QoL of patients with HNC include clinical and sociodemographic characteristics [5], but also a variety of psychological factors, including depression, anxiety, alcohol abuse, illness perceptions and coping strategies [4,6]. Evaluation of factors affecting QoL in patients with HNC continues to be of interest, as a recent 10-year follow-up study documented that QoL in the first year after diagnosis can predict survival $[7,8]$.

Most studies investigating the association between illness perceptions, emotional distress, coping strategies and QoL in head and neck cancer patients have been cross-sectional, based on a single assessment, either immediately after diagnosis [4,9] or at follow-up [10]. As pointed out by a recent systematic review [1], the directionality of the correlations between psychological distress and coping strategies in head and neck cancer patients is not easily understood. Due to the insufficient data, it is necessary to carry out prospective design studies which will focus, mainly, on the definition of temporal patterns of psychosocial functioning that may affect the associations between the aforementioned variables and the quality of life in head and neck cancer patients. Additionally, among studies there are conflicting findings about the association between active coping style aimed at direct change and adjust- 
ment to the illness and mental distress experienced by patients with head and neck cancer $[1,11,12]$. The findings that appeared to be most consistent are those suggesting that avoidance and disengagement mechanisms are associated with increased mental distress in patients with head and neck cancer. To the best of our knowledge there are few prospective studies that have assessed the psychological distress, illness perceptions and coping strategies and their impact on quality of life in patients with head and neck cancer and even fewer in patients with head and neck cancer undergoing radiotherapy [13]. For all the aforementioned reasons the aim of the present study was to prospectively assess the psychological distress, illness perceptions and coping strategies adopted by patients with HNC undergoing radiotherapy at two time points, in a pre- and a post radiation period and to investigate their predictive value on QoL in a short-term after RT.

\section{MATERIAL AND METHODS}

\section{Participants}

The sample consisted of the first 50 consecutive patients diagnosed with primary HNC who started radiotherapy during the study period and agreed to participate. All patients were recruited from the same Radiation Oncology Department of a University Hospital. Exclusion criteria were age $>75$ years, metastatic disease, co-administration of chemotherapy, a diagnosis of dementia, abuse of substances, mental illness, previous participation in psycho-educational intervention, and inability to complete the questionnaires for any reason. From the patients who fulfilled the criteria and were invited to participate $(\mathrm{N}=60), 6$ patients were excluded due to the missing data, 4 declined to participate and 50 agreed (response rate 83.3\%).

\section{Procedure and Study Instruments}

The study was conducted in accordance with the Ethical Standards as formulated in the World Medical Association Helsinki Declaration (2002) and its later amendments, and an IRB statement that IRB approval was unnecessary, was received. The participants were informed about the study objectives and data confidentiality and signed a consent form according to the aforementioned principles.

Assessment was made at two time points, the first prior to the simulation appointment and the onset of radiotherapy (T1) and the second at the first follow-up, 3 months after completion of radiotherapy (T2). The choice of the follow-up point time at 3 months after the completion of radiotherapy was based on the following elements: a. it was the first scheduled follow-up visit at the specific Radiation Oncology Department and b. it was considered as a sufficient period for the remission of the acute adverse effects of RT and, consequently, for the reduction of their effects on the quality of life of the patients. All the medical information was collected by one radiation oncologist and all the psychological assessments were conducted by one clinical psychologist. The study was prospective, questionnaire based, with repeated measures, and the patients were asked to complete the following research tools.

\section{The Hospital Anxiety and Depression Scale (HADS)}

HADS consists of 14 items and aims to measure symptoms of anxiety (HADS-A) and depression (HADS-D) [14]. The answers are given on a Likert scale of 0-3 and the score of each of the two sub-scales ranges from 0 to 21, with the higher value corresponding to more frequent and severe symptoms. Value categorization is as follows: 0-7: normal, 8-10: mild, 11-14: moderate, 15-21: severe. The questionnaire has been translated and validated in the Greek language [15].

\section{The Revised IIIness Perception Questionnaire (IPQ-R)}

IPQ-R consists of 38 items and provides a quantitative assessment of the nature and strength of perceptions in nine dimensions [16]: illness identity (perception of symptoms related to the illness), acute/chronic timeline (perceived duration of the illness), timeline cyclical, consequences, personal control (self-efficacy beliefs), treatment control (treatment expectations), illness coher- ence (the degree of overall coherent understanding of the illness), emotional representations (symptoms of anxiety and depression experienced as result of the illness) and causal attributions of the illness: emotional (e.g., stress), behavioural (e.g., smoking) and external causes (e.g., chance) and risk factors (e.g., immune system). Responses are given on a 5-point Likert scale (ranging from "Absolutely disagree" to "Absolutely agree"). Higher values are indicative of a stronger perception. The item on the perceived cause of the illness is open-ended, requesting the participants to rank the three most important causal factors. The version used was the form of the questionnaire validated in the Greek language [17].

\section{The Brief COPE Inventory}

The Brief COPE Inventory for assessing coping strategies is the modified version of the original COPE Inventory [18], and it has been validated in Greek [19]. It includes 28 items corresponding to 14 scales: self-distraction, active coping, denial, substance use, use of emotional support, instrumental support, behavioural disengagement, venting, positive reframing, planning, humour, acceptance, religion, self-blame. The instructions were modified in order for participants to report what they did when they specifically experienced their disease (cancer) or treatment side-effects. The answers range from 1 "I do not do it at all", to 4 "I do it a lot". Higher scores correspond to more frequent use of each strategy.

\section{EORTC-QLQ-C30 \& EORTC-QLQ-H\&N35}

The European Organization for Research and Treatment of Cancer Quality of Life Questionnaire (EORTC-QLQ-C30) and the disease-specific questionnaire of EORTC for HNC (EORTC-QLQ-H\&N35) were also administered [20,21]. The Greek versions of both questionnaires have been provided by the official website of EORTC study tools (https://qol.eortc.org). The EORTC-QLQ-C30 consists of 30 items classified into 5 domains: Physical, Role, Emotional, Cognitive and Social functioning, and the Global Health Status (GHS), while there are certain single items of symptoms. EORTC-QLQ-H\&N35 consists of 35 items that correspond to seven domains: pain, swallowing, senses, speech, social eating, social contact, and sexuality. In addition, 11 independent items refer to specific physical symptoms (e.g., teeth problems, dry mouth). In both questionnaires, responses are given on Likert 4 and 7-point scales, and the scores ranged from 0 to 100 . On the symptom items, the highest score corresponds to a stronger intensity, while on the functional scales, the highest score corresponds to a better quality of life. The symptoms sum score provides a generalizing score of QoL (H\&N sum score).

\section{Statistical Analysis}

Mean and standard deviation (SD) were used to describe the scores on the scales used in the study. Frequencies and percentages were used for categorical variables such as demographic data and stage of cancer. A general linear model of repeated measures was applied to detect statistically significant differences between the scores at the two study periods, T1 and T2 on the various scales of EORTC-QLQ-C30, H\&N35, HADS, IPQ-R and Brief COPE. In order to investigate predictive factors of QoL, the effects of the HADS, IPQ-R and Brief COPE scores at $\mathrm{T} 1$ and the demographic and clinical characteristics were examined in each regression model hierarchically, after examining the univariate effect of each one separately using correlation analyses or mean comparison statistics. Specifically, a blockwise forward method was adopted, entering the dimensions of the IPQ-R, HADS and Brief COPE in this specific order. The model with statistically significant outcomes was then tested for changes after controlling for the effect of demographic characteristics such as age, gender, and educational level, and for smoking status, alcohol consumption, disease stage, previous chemotherapy, and surgery. The forward blockwise model was adopted separately for each of the domains of the EORTC-QLQ-C30 and the H\&N35 sum score in order to examine the outcomes in a hierarchical manner in terms of interest, but also to assure the validity, as the small number of participants would lead to unreliable models if the simultaneous enter approach had been chosen. Statistical significance was set at 0.05 for all tests. Analysis was conducted with the use of SPSS v22.0. 


\section{ORIGINAL}

Table 1. Sociodemographic and Clinical Characteristics of the Study Patients With Head and Neck Cancer $(\mathrm{N}=50)$

\begin{tabular}{|c|c|}
\hline Variable & Value \\
\hline Age (Mean \pm SD) (Range) & $62.9 \pm 8.3(38-74)$ \\
\hline \multicolumn{2}{|l|}{ Gender $(\mathrm{N}, \%)$} \\
\hline Female & $7(14)$ \\
\hline Male & $43(86)$ \\
\hline \multicolumn{2}{|l|}{ Family status (N, \%) } \\
\hline Single & $3(6)$ \\
\hline Married/domestic partnership & $39(78)$ \\
\hline Divorced/separated & $3(6)$ \\
\hline Widowed & $5(10)$ \\
\hline \multicolumn{2}{|l|}{ Educational level (N, \%) } \\
\hline Primary school & $31(62)$ \\
\hline High school & $11(22)$ \\
\hline University degree & $8(16)$ \\
\hline Disease duration (Months, Mean \pm SD) & $13.42 \pm 7.74$ \\
\hline \multicolumn{2}{|l|}{ AJCC Stage of cancer ( $\mathrm{N}, \%)$} \\
\hline I & $13(26)$ \\
\hline$\|$ & $11(22)$ \\
\hline III & $11(22)$ \\
\hline IV & $15(30)$ \\
\hline \multicolumn{2}{|l|}{ Cancer site ( $\mathrm{N}, \%)$} \\
\hline Buccal mucosa & $3(6)$ \\
\hline Maxillary & $6(12)$ \\
\hline Lingual & $3(6)$ \\
\hline Mouth floor & $7(14)$ \\
\hline Oropharynx & $9(18)$ \\
\hline Larynx & $20(40)$ \\
\hline Salivary Gland & $2(4)$ \\
\hline \multicolumn{2}{|l|}{ Surgery (N, \%) } \\
\hline Yes & $29(58)$ \\
\hline No & $21(42)$ \\
\hline \multicolumn{2}{|l|}{ Chemotherapy (N, \%) } \\
\hline Yes & $23(46)$ \\
\hline No & $27(54)$ \\
\hline \multicolumn{2}{|l|}{ Type of radiotherapy $(\mathrm{N}, \%)$} \\
\hline Postoperative & $21(42)$ \\
\hline Radical & $29(58)$ \\
\hline \multicolumn{2}{|l|}{ Smoking (N, \%) } \\
\hline Yes & $37(74)$ \\
\hline No & $13(26)$ \\
\hline No. of cigarettes per day & $32.2 \pm 20.9$ \\
\hline Years of smoking (Mean $\pm S D$ ) (Range) & $25.59 \pm 14.20(1-55)$ \\
\hline \multicolumn{2}{|l|}{ Alcohol consumption ( $\mathrm{N}, \%)$} \\
\hline Yes & $35(70)$ \\
\hline No & $15(30)$ \\
\hline
\end{tabular}

AJCC, American Joint Committee on Cancer; SD, standard deviation.
Table 2. Scores on Quality of Life and Anxiety and Depression Questionnaires of Patients With Head and Neck Cancer ( $N=50)$, Before (T1) and 3 Months After (T2) Radiotherapy

\begin{tabular}{|c|c|c|}
\hline Questionnaire & $\mathrm{T} 1($ Mean $\pm \mathrm{SD})$ & $\mathrm{T} 2($ Mean $\pm \mathrm{SD})$ \\
\hline \multicolumn{3}{|l|}{ EORTC-QLQ-C30 } \\
\hline Global Health Status (GHS) & $54.42 \pm 25.06$ & $57.48 \pm 24.13$ \\
\hline Physical Functioning & $76.27 \pm 17.72$ & $76.87 \pm 23.96$ \\
\hline Role Functioning & $68.00 \pm 33.64$ & $68.03 \pm 29.82$ \\
\hline Emotional Functioning & $69.67 \pm 25.52$ & $73.30 \pm 23.45$ \\
\hline Cognitive Functioning & $83.67 \pm 22.21$ & $86.39 \pm 20.03$ \\
\hline Social Functioning & $80.00 \pm 26.94$ & $81.29 \pm 22.21$ \\
\hline Fatigue & $32.00 \pm 26.24$ & $32.88 \pm 25.05$ \\
\hline Nausea/vomiting & $5.67 \pm 20.37$ & $8.50 \pm 16.00$ \\
\hline Pain & $17.00 \pm 20.62$ & $21.77 \pm 23.13$ \\
\hline Dyspnoea & $32.67 \pm 31.22$ & $30.61 \pm 33.22$ \\
\hline Insomnia & $26.67 \pm 32.99$ & $21.77 \pm 26.83$ \\
\hline Appetite loss & $15.33 \pm 24.48$ & $25.17 \pm 27.66$ \\
\hline Constipation & $15.33 \pm 27.11$ & $10.20 \pm 15.52$ \\
\hline Diarrhoea & $6.00 \pm 12.94$ & $2.04 \pm 8.07$ \\
\hline Financial problems & $46.00 \pm 33.6$ & $42.86 \pm 34.69$ \\
\hline \multicolumn{3}{|l|}{ EORTC QLQ H\&N35 } \\
\hline Pain & $19.67 \pm 19.33$ & $22.28 \pm 21.41$ \\
\hline Swallowing & $18.39 \pm 22.01$ & $22.45 \pm 23.95$ \\
\hline Senses & $27.33 \pm 32.60$ & $26.53 \pm 20.39$ \\
\hline Speech & $30.89 \pm 32.16$ & $24.04 \pm 26.09$ \\
\hline Social eating & $17.17 \pm 22.74$ & $24.83 \pm 23.90$ \\
\hline Social contact & $15.07 \pm 23.20$ & $16.73 \pm 23.46$ \\
\hline Sexuality & $40.48 \pm 39.09$ & $42.01 \pm 35.89$ \\
\hline Teeth & $24.67 \pm 36.15$ & $25.17 \pm 25.94$ \\
\hline Opening mouth & $20.00 \pm 27.77$ & $17.69 \pm 26.44$ \\
\hline Dry mouth & $26.67 \pm 26.08$ & $41.5 \pm 28.49 *$ \\
\hline Sticky saliva & $27.33 \pm 31.36$ & $38.78 \pm 30.69$ \\
\hline Coughing & $22.00 \pm 27.45$ & $17.01 \pm 26.46$ \\
\hline Feeling ill & $18.00 \pm 24.48$ & $27.21 \pm 28.60$ \\
\hline H\&N 35 Sum Score & $22.52 \pm 13.85$ & $24.45 \pm 14.46$ \\
\hline HADS-A & $6.44 \pm 5.29$ & $5.44 \pm 4.28$ \\
\hline HADS-D & $6.56 \pm 4.85$ & $5.62 \pm 4.29$ \\
\hline
\end{tabular}

EORTC QLQ-C30, European Organization for Research and Treatment of Cancer Quality of Life Questionnaire; EORTC QLQ H\&N35, disease-specific questionnaire for head and neck cancer; HADS: Hospital Anxiety and Depression Scale (A: anxiety, D: depression); SD, standard deviation. ${ }^{*} \mathrm{P}<0.05$ 
Table 3. Scores on the Questionnaires IPQ-R and Brief Cope of Patients With Head and Neck Cancer ( $N=50$ ) Before (T1) and 3 Months After (T2) Radiotherapy

\begin{tabular}{|c|c|c|}
\hline Questionnaire & T1 (Mean \pm SD) & $\mathrm{T} 2($ Mean \pm SD) \\
\hline \multicolumn{3}{|l|}{ IPQ-R } \\
\hline Identity & $10.21 \pm 1.74$ & $11.03 \pm 1.16$ \\
\hline Timeline acute/chronic & $20.80 \pm 2.44$ & $21.13 \pm 2.76$ \\
\hline Timeline cyclical & $13.61 \pm 2.03$ & $14.10 \pm 2.14$ \\
\hline Consequences & $20.49 \pm 2.50$ & $19.78 \pm 1.96$ \\
\hline Personal control & $18.67 \pm 2.38$ & $18.57 \pm 2.18$ \\
\hline Treatment control & $16.29 \pm 2.41$ & $15.80 \pm 2.29$ \\
\hline Illness coherence & $14.02 \pm 2.21$ & $13.84 \pm 1.66$ \\
\hline Emotional representations & $18.76 \pm 1.86$ & $18.92 \pm 1.81$ \\
\hline \multicolumn{3}{|l|}{ Causes } \\
\hline Emotional cause & $2.78 \pm 0.79$ & $2.73 \pm 0.69$ \\
\hline Behavioural cause & $3.00 \pm 0.69$ & $3.45 \pm 0.45^{\star \star \star}$ \\
\hline Risk factors & $3.08 \pm 0.67$ & $3.49 \pm 0.52^{\star \star}$ \\
\hline External & $3.24 \pm 1.18$ & $2.90 \pm 1.31$ \\
\hline \multicolumn{3}{|l|}{ Brief COPE } \\
\hline Self-distraction & $2.21 \pm 1.04$ & $2.04 \pm 1.36$ \\
\hline Active coping & $2.30 \pm 0.88$ & $2.51 \pm 1.08$ \\
\hline Denial & $1.97 \pm 0.97$ & $2.17 \pm 1.03$ \\
\hline Substance use & $1.32 \pm 0.71$ & $1.36 \pm 0.70$ \\
\hline Emotional support & $2.32 \pm 1.00$ & $2.36 \pm 0.96$ \\
\hline Instrumental support & $2.15 \pm 1.03$ & $2.25 \pm 1.11$ \\
\hline Behavioural disengagement & $1.55 \pm 0.85$ & $1.58 \pm 0.88$ \\
\hline Venting & $2.15 \pm 0.92$ & $2.09 \pm 0.99$ \\
\hline Positive reframing & $2.42 \pm 0.83$ & $2.23 \pm 0.91$ \\
\hline Planning & $2.48 \pm 0.93$ & $2.49 \pm 0.91$ \\
\hline Humour & $1.64 \pm 0.90$ & $1.62 \pm 0.95$ \\
\hline Acceptance & $2.86 \pm 0.96$ & $2.88 \pm 1.00$ \\
\hline Religion & $2.05 \pm 0.99$ & $2.08 \pm 0.94$ \\
\hline Self-blame & $2.25 \pm 1.09$ & $2.25 \pm 1.09$ \\
\hline
\end{tabular}

IPQ-R, Revised IIIness Perception Questionnaire; SD, standard deviation. ** $\mathrm{P}<0.05 ;{ }^{*} * \star \mathrm{P}<0.001$

\section{RESULTS}

\section{Characteristics of the Sample}

The characteristics of the study sample are shown in Table 1. The mean age of the participants was $62.9 \pm 8.3$ years (range 38-74), with the majority being of men (86\%), married (78\%), of low educational level (62\% were primary school graduates), smokers (74\%) and alcohol consumers (70\%). Regarding the clinical features, $58 \%$ of the sample had undergone surgery and the majority underwent radical radiotherapy (58\%) (Table1).

\section{EORTC-QLQ-C30 and H\&N35}

On comparison of the scores on EORTC-QLQ-C30 and H\&N35 at T1 and T2 no significant difference was found in either the main domains or in the symptoms, apart from the symptom of dry mouth, which was aggravated after radiotherapy ( $P=0.015)$. The mean scores on some of the items (e.g., sticky saliva, a general sense of "feeling ill", and difficulties with social eating) were higher at T2 than T1, but not to a statistically significant degree (Table 2).

\section{HADS}

In general, low levels of anxiety and depression were elicited (HADS $<8$ ) at both time points, with no significant difference between the T1 and the T2 measurements (Table 2).

\section{IPQ-R and Brief COPE}

Regarding IPQ-R, the strongest beliefs at both T1 and T2 concerned the chronic timeline of the illness, its negative consequences and the emotional representations. Comparison of the mean scores on IPQ-R at T1 and T2 to explore possible changes in the patients' perceptions showed change only regarding the perceived causes of their illness. Specifically, at T2 there was a significant increase in the belief that the illness was due to suppression of the immune system $(p<0.001)$ and to behavioural factors (e.g., smoking, alcohol) $(p<0.001)$. Concerning the Brief COPE, no significant difference was demonstrated between the scores at T1 and T2 (Table 3).

\section{Quality of Life Associations}

No significant correlations were shown between the socio-demographic and clinical characteristics of the patients and the domains of the EORTC-QLQ-C30 and the H\&N35. Significant correlations were observed between HADS subscales, Brief COPE and IPQ-R, as assessed at T1, and the domains of EORTC-QLQ-C30 and H\&N sum score as assessed at T2, as shown in detail in Table 4.

The main aim of this study was to identify the variables that, when measured at T1, could predict QoL outcomes at T2. On hierarchical regression analysis, it became evident that depression at T1 was a significant predictor of Physical, Role and Social Functioning (EORTC-QLQ-C30). A higher HADS-D at $\mathrm{T} 1$ was associated with lower Physical (beta $=-1.533, \mathrm{P}=0.025$ ), Role (beta $=-2.054, \mathrm{P}=0.019$ ) and Social Functioning (beta $=-1.419, \mathrm{P}=0.019)$ at $\mathrm{T} 2$. In addition, a higher score on Behavioural disengagement (Brief COPE) was associated with lower scores on Physical (beta $=-9.572, P=0.013$ ), Emotional (beta $=-11.341, P=0.003$ ), Cognitive (beta $=-6.442, P=0.042$ ) and Social Functioning (beta $=-6.791, \mathrm{P}=0.040$ ). GHS at T2 was closely associated with beliefs about Treatment controllability (IPQ-R) (beta $=3.832, \mathrm{P}=0.007$ ) and Substance use (Brief COPE) (beta $=-11.523, P=0.015$ ) at T1. Two dimensions of the IPQ-R were found to be associated with H\&N sum score at follow-up. Specifically, stronger Emotional representations and weaker beliefs about IIIness coherence at T1 were associated with higher H\&N35 sum score at T2 (beta = 2.401, $P=0.024$ and beta $=-1.922, P=0.032$, respectively). Finally, stronger perceived control (IPQ-R) at T1 was associated with higher Social functioning at T2 (beta $=-2.931, P=0.011$ ) (Table 5). In analysis, all outcomes were adjusted for age, gender, education, smoking status, alcohol consumption, disease stage, surgery and previous chemotherapy.

\section{DISCUSSION}

The present study assessed psychological distress, illness perceptions, coping strategies and QoL in patients with H\&N cancer before and after radiotherapy, and investigated the predictive role of psychological distress, illness perceptions and coping strategies on various dimensions of QoL three months after radiotherapy.

With regard to QoL, little change was detected between the measurements before and 3 months after radiotherapy, except in dry mouth, a symptom that was significantly worse after radiotherapy. A well-known side-effect of irradiation is mucosal dryness, along with muscle atrophy and erythema. Patients experience pain, swallowing disorders and voice problems soon after radiotherapy, with a resulting marked decrease in overall QoL [22]. In addition, it was found that certain symptoms were slightly more intense after radiotherapy, but a literature search to identify minimally important differences (MID) in QoL scores [23] revealed no reports on patients with HNC, and no further interpretation of the changes in mean scores, in terms of clinical meaningfulness, is possible. 


\section{ORIGINAL}

Table 4. Zero Order Correlations Between Scores on HADS, IPQ-R and Brief COPE Questionnaires Before Radiotherapy (T1) and EORTC-QLQ-C30 and H\&N 35 (Sum Score), Three Months After Radiotherapy (T2) In Patients With Head and Neck Cancer ( $=50)$

\begin{tabular}{|c|c|c|c|c|c|c|c|}
\hline Questionnaire & GHS & PF & $\mathrm{RF}$ & $\mathrm{EF}$ & $C D$ & SF & H\&N 35 \\
\hline \multicolumn{8}{|l|}{ HADS } \\
\hline HADS-A & -0.219 & -0.174 & -0.198 & $-0.354 *$ & -0.176 & $-0.453 * *$ & $0.346^{*}$ \\
\hline HADS-D & $-0.365^{\star *}$ & $-0.443^{* *}$ & $-0.335^{*}$ & $-0.347^{*}$ & $-0.328 *$ & $-0.496 * *$ & $0.317^{*}$ \\
\hline \multicolumn{8}{|l|}{ IPQ-R } \\
\hline Identity & 0.053 & 0.104 & 0.132 & 0.140 & 0.090 & 0.204 & -0.119 \\
\hline Timeline (acute/chronic) & -0.206 & -0.127 & -0.02 & -0.2 & $-0.342 *$ & -0.04 & 0.151 \\
\hline Timeline cyclical & -0.194 & 0.003 & -0.071 & 0.001 & -0.14 & $-0 . .079$ & 0.19 \\
\hline Consequences & 0.016 & -0.113 & -0.028 & $-0.298^{*}$ & -0.134 & -0.119 & 0.171 \\
\hline Personal control & 0.126 & 0.098 & 0.086 & 0.268 & 0.153 & $0.446 * *$ & $-0.303^{*}$ \\
\hline Treatment control & $0.293^{*}$ & 0.246 & 0.245 & 0.141 & $0.34^{*}$ & 0.264 & -0.218 \\
\hline Illness coherence & 0.238 & 0.156 & 0.126 & $0.315^{*}$ & 0.21 & $0.326^{*}$ & $-0.347 *$ \\
\hline Emotional representations & -0.174 & -0.167 & -0.175 & $-0.323^{*}$ & -0.193 & -0.205 & $0.36 *$ \\
\hline \multicolumn{8}{|l|}{ Brief COPE } \\
\hline Self distraction & 0.168 & 0.147 & 0.097 & -0.068 & -0.087 & 0.01 & -0.014 \\
\hline Active coping & $0.352^{*}$ & $0.364^{* *}$ & $0.286^{*}$ & 0.15 & 0.075 & 0.093 & -0.197 \\
\hline Denial & -0.255 & $-0.353^{*}$ & $-0.347^{*}$ & $-0.423^{* *}$ & $-0.317^{*}$ & $-0.428^{* *}$ & $0.403^{* *}$ \\
\hline Substance use & -0.247 & -0.196 & -0.254 & $-0.421 * *$ & -0.058 & -0.281 & 0.275 \\
\hline Emotional support & 0.089 & -0.101 & 0.026 & -0.053 & 0.13 & -0.032 & 0.167 \\
\hline Instrumental support & $0.337^{\star}$ & 0.085 & 0.173 & -0.075 & 0.153 & 0.133 & 0.104 \\
\hline Behavioural disengagement & -0.223 & $-0.463^{* *}$ & -0.26 & $-0.473^{* *}$ & -0.291 * & $-0.439 * *$ & 0.209 \\
\hline Venting & -0.014 & -0.182 & 0.018 & $-0.415^{\star \star}$ & -0.126 & -0.204 & $0.315^{*}$ \\
\hline Positive reframing & 0.18 & 0.176 & 0.025 & 0.177 & 0.005 & 0.139 & -0.038 \\
\hline Planning & 0.208 & 0.208 & 0.081 & 0.038 & -0.015 & -0.052 & 0.026 \\
\hline Humour & -0.12 & 0.106 & -0.071 & -0.158 & -0.053 & -0.173 & 0.058 \\
\hline Acceptance & 0.105 & -0.052 & -0.094 & -0.173 & -0.075 & -0.094 & 0.19 \\
\hline Religion & -0.092 & -0.041 & -0.112 & -0.233 & -0.084 & -0.058 & 0.251 \\
\hline Self blame & -0.073 & -0.07 & -0.182 & -0.215 & -0.237 & -0.269 & 0.229 \\
\hline
\end{tabular}

CF, Cognitive Functioning; EORTC QLQ-C30, European Organization for Research and Treatment of Cancer Quality of Life questionnaire; EF, Emotional Functioning; GHS, Global Health Status; HADS, Hospital Anxiety and Depression Scale (A: anxiety, D: depression); H\&N35, sum score of EORTC QLQ H\&N35 disease-specific questionnaire for head and neck cancer; IPQ-R, Revised IIIness Perceptions Questionnaire; PF, Physical Functioning; RF, Role Functioning; SF, Social Functioning.

Questionnaire; $P F$, Physical Func
$* \mathrm{P}<0.05 ; * \star \mathrm{P}<0.01 ; * \star * \mathrm{P}<0.001$ 
Table 5. Statistically Significant Effects of Factors Measured Before Radiotherapy (T1) On Quality of Life According to EORTC QLQ-C30 and H\&N 35 Three Months After Radiotherapy (T2) In Patients With Head and Neck Cancer ( $N=50)$

\begin{tabular}{|c|c|c|c|}
\hline \multirow[b]{2}{*}{$R^{2}=0.163$} & \multicolumn{3}{|c|}{ Global health status } \\
\hline & Beta & $\mathrm{t}$ & Significance \\
\hline Treatment Control (IPQ-R) & 3.832 & 2.804 & 0.007 \\
\hline \multirow[t]{2}{*}{ Substance Use (Brief COPE) } & -11.523 & -2.533 & 0.015 \\
\hline & \multicolumn{3}{|c|}{ Physical functioning } \\
\hline$R^{2}=0.266$ & Beta & $\mathrm{t}$ & Significance \\
\hline HADS-D & -1.533 & -2.232 & 0.025 \\
\hline \multirow[t]{2}{*}{ Behavioural Disengagement (Brief COPE) } & -9.572 & -2.572 & 0.013 \\
\hline & \multicolumn{3}{|c|}{ Role functioning } \\
\hline$R^{2}=0.093$ & Beta & $\mathrm{t}$ & Significance \\
\hline \multirow[t]{2}{*}{ HADS-D } & -2.054 & -2.434 & 0.019 \\
\hline & \multicolumn{3}{|c|}{ Emotional functioning } \\
\hline $\mathrm{R}^{2}=0.234$ & Beta & $\mathrm{t}$ & Significance \\
\hline \multirow[t]{2}{*}{ Behavioral Disengagement (Brief COPE) } & -11.341 & -3.185 & 0.003 \\
\hline & \multicolumn{3}{|c|}{ Cognitive functioning } \\
\hline$R^{2}=0.157$ & Beta & $\mathrm{T}$ & Significance \\
\hline Treatment Control (IPQ-R) & 2.726 & 2.478 & 0.017 \\
\hline \multirow[t]{2}{*}{ Behavioural Disengagement (Brief COPE) } & -6.442 & -2.089 & 0.042 \\
\hline & \multicolumn{3}{|c|}{ Social functioning } \\
\hline$R^{2}=0.370$ & Beta & $\mathrm{T}$ & Significance \\
\hline Personal Control (IPQ-R) & 2.931 & 2.635 & 0.011 \\
\hline HADS-D & -1.419 & -2.442 & 0.019 \\
\hline \multirow[t]{2}{*}{ Behavioural Disengagement (Brief COPE) } & -6.791 & -2.116 & 0.040 \\
\hline & \multicolumn{3}{|c|}{$\mathrm{H} \& \mathrm{~N} 35$ sum score } \\
\hline$R^{2}=0.179$ & Beta & $\mathrm{T}$ & Significance \\
\hline Emotional Representations (IPQ-R) & 2.401 & 2.333 & 0.024 \\
\hline Illness Coherence (IPQ-R) & -1.922 & -2.216 & 0.032 \\
\hline
\end{tabular}

Beta coefficient and P values for the quality-of-life outcomes after Hierarchical Regression Analysis. Results are adjusted for age, gender, educational level, smoking status, alcohol consumption, disease stage, surgery, and previous chemotherapy. Brief COPE, Brief COPE Inventory; EORTC QLQ-C30, European Organization for Research and Treatment of Cancer Quality of Life questionnaire; HADS, Hospital Anxiety and Depression Scale (A: anxiety, D: depression); H\&N 35, EORTC disease specific questionnaire for head and neck cancer; IPQ-R, Revised IIIness Perceptions Questionnaire.

Regarding psychological distress, the study findings are in line with previous reports $[24,25]$ as low mean values in anxiety and depression levels ( $<8$ on HADS-A and HADS-D) were found. Studying the association between HADS levels at T1 and QoL outcomes at T2, it became evident that HADS-D at T1 affected Physical, Role and Social Functioning at T2. Specifically, a higher HADS-D score before radiotherapy was associated with lower scores on Physical, Role and Social Functioning three months after radiotherapy. Depression has already been identified as an important predictor of the survival and the QoL of patients with HNC $[26,27]$. Patients with HNC who show high levels of anxiety and depression, more often react with a sense of hopelessness/ helplessness and constant preoccupation with the illness, resulting in a deterioration of QoL $[28,29]$. Reported rates of depression and anxiety in patients with HNC range from $20 \%$ to $40 \%$, and both cross-sectional and long-term studies have shown that psychological distress is predictor, not only of QoL but also of survival [30].

With regard to the illness perceptions (IPQ-R) and coping strategies (Brief COPE), no significant differences were detected between the measurements before and after radiotherapy. This may be due to the fact that certain strategies had already been adopted before the initial study assessment, i.e., during the interim period between the diagnosis and the start of radiotherapy. Longitudinal studies [31,32] and a systematic review [33] have concluded that, without specific intervention, illness perceptions and coping strategies show little modification over time even when they are assessed shortly after the diagnosis of a life-threatening illness.

In this study, Behavioural Disengagement (Brief COPE) at T1, as a coping strategy characterized by a passive reaction and a tendency to give up the management of external demands, appeared to affect the domains of Physical, Emotional, Cognitive and Social Functioning at T2. A large body of evidence indicates that denial, behavioural disengagement and self-blame at the initial post-diagnosis assessment are prognostic factors of poor QoL [33,34]. In addition, Substance use as a coping strategy at T1 appeared to be correlated with impairment of the overall health status (GHS) at T2. Unfortunately, one quarter of patients with HNC have been observed to continue to drink or smoke after the diagnosis of HNC [35]. Several factors may contribute to continued use, including psychological distress, lack of motivation and individual perceptions of risk and causal attributions. Several studies have reported that patients with HNC are more likely to rely on avoidant or other maladaptive strategies that are significantly associated with poorer QoL [36,37] and to adopt different strategies in different phases of the treatment [12,38]. Specifically, patients under treatment or in the less-than-6-month post-treatment phase, more frequently adopt such strategies as denial, behavioural disengagement and suppression, than patients assessed either before the start of treatment or at follow-up longer than 6 months after completion of the treatment. When people are defending themselves against a difficult situation, they tend to avoid experiences and unpleasant feelings. In the case of long-term illnesses, patients with strong perceptions of chronic timeline and multiple consequences may adopt denial/avoidance coping mechanisms, according to which they operate as if they did not have the problem; this mechanism may become more intense if they believe that certain behavioural factors have contributed to the onset of the disease [39]. Age, gender, low educational level and previous alcohol and tobacco use have been proposed to explain the adoption of these strategies [40].

In addition, it has been argued that the coping strategies adopted by the patients with HNC vary according to their levels of anxiety and depression [36]. A recent systematic review [1] highlighted the moderate to high correlation between emotional disengagement and psychological distress observed in a total of 12 relevant studies. Strategies that minimize threatening thoughts can be useful in reducing stress at critical stages of the disease, and be associated with positive adjustment [41].

Regarding illness perceptions (IPQ-R), it was found that a stronger perception of controllability by treatment at $\mathrm{T} 1$ was associated with better overall health status (GHS) at T2. In addition, perceived personal controllability at T1 was correlated positively with Social functioning and negatively with the H\&N35 sum score at T2. Previous studies concluded that efficacy beliefs and perceived curability should be taken into account, as they appear to have a significant impact on the psychological well-being and QoL of patients with malignancy $[42,43]$.

Moreover, Emotional Representations (IPQ-R) at T1, i.e., the extent to which the illness is believed to result in psychological distress symptoms, was positively correlated with the H\&N35 sum score at T2. This means that the more strongly the patient believes that the illness will affect their emotional well-being, the more severe are the symptoms, and, consequently, the poorer is their QoL. The H\&N35 sum score at T2 was also correlated with the Illness coherence (IPQ-R), which reflects the individual's overall coherent understanding of the illness. Thus, when patients fail to understand the illness in a coherent way, their experience of symptoms is more intense. It had already been shown that various illness perceptions are correlated with certain dimensions of QoL, and that those patients who focus their attention on the symptoms, experience intense fear of relapse, exhibit emotional responses to their illness and self-blame, and have poorer QoL. In particular, Emotional Representations and perceived Treatment Controllability are consistently reported to be correlated with the outcome of the illness, as demonstrated by a recent 
meta-analysis [44]. Another meta-analysis, on the relationship between illness perceptions and emotional distress of patients in a variety of physical illnesses [33], concluded that patients experiencing a strong sense of hopelessness were more likely to have higher levels of anxiety and depression, in contrast to patients with positive perceptions of the effects of the illness and an increased sense of external control. However, a high level of heterogeneity is observed in the association of illness perceptions with health outcomes in cancer patients. This is probably due to the multiple moderators related to the diagnosis and treatment, and further investigation is recommended on the causal effects of illness perceptions on psychological distress, coping and QoL [45].

\section{Suggestions and Limitations}

Regular psychological assessment and early inclusion in a psychotherapy program is required for patients to achieve better adaptation to illness, with cognitive restructuring, adoption of effective coping strategies and decrease of the psychological burden [43]. Interventions focusing on the enhancement of beliefs about controllability appear to be more effective in improving emotional well-being, preventing chronic psychopathology and improving QoL $[31,46]$. It is of note that, although there are concerns about the participation rate of patients with HNC in psychological interventions because of low socio-economic level, they are reported to be well-involved in psycho-educational programs and cognitive behavioural therapy (CBT) [47].

The present study has some limitations and cautious interpretations of the results are needed. Firstly, the sample was small, as, given the population of the district where the study was conducted and the incidence of $\mathrm{H} \& \mathrm{~N}$ cancer, it was possible only 50 participants to be included during the study period. Secondly, in the present study, only patients undergoing radiotherapy participated while patients with a complete removal of tumor via surgical intervention or other type of treatment were not included, a fact that limits the possibility of generalizing the results. Thirdly, the time that had elapsed between the diagnosis and the initial assessment of patients is an additional limitation, as it was not possible to evaluate prior modifications of illness perceptions and coping strategies, at what time these may had been occurred and what factors contributed. Finally, the heterogeneity of treatment which the patients had undergone prior to joining the study is a factor that restricts the generalizability of the results.

Despite the aforementioned limitations, some strengths of the study are its prospective design, the use of the extended version of the IPQ, i.e., the IPQ-R, which assesses more fully the basic dimensions of the questionnaire, and the focussing on the role of certain psychological factors on the QoL of HNC patients undergoing radiotherapy, a subject that has not been adequately studied.

It would be important for future research to be conducted with a larger sample, starting assessment immediately after diagnosis, and to conduct a long-term comparative study of the impact of psycho-educational intervention and the enhancement of effective coping strategies on the QoL of patients with HNC undergoing radiation therapy.

\section{CONCLUSIONS}

In conclusion, depressive symptoms before the start of radiotherapy are predictors of impairment of the QoL of patients with HNC three months after the completion of treatment. Behavioral Disengagement as a coping strategy and strong beliefs about emotional representations of the illness may also predict impairment in certain domains of the QoL of patients with HNC at follow-up, while belief in treatment controllability may enhance QoL. The identification and the understanding of the depressive symptoms of patients, their beliefs about their illness and their avoidance coping strategies can provide the basis for timely implementation of appropriate intervention aimed at improving the QoL of patients with HNC undergoing radiation therapy.

\section{ARTICLE INFORMATION}

*Correspondence: Vassiliki Siafaka, MSc, PhD, Department of Speech and Language Therapy, School of Health Sciences, University of loannina, $4^{\text {th }} \mathrm{Km}$ National Road loanninaAthens, loannina 45500, Greece. Email: siafaka@uoi.gr; vsiafaka@gmail.com

Received: Aug. 04, 2020; Accepted: Oct. 15, 2020; Published:Jul. 05, 2021

DOI: 10.24983/scitemed.aohns.2021.00143

Author Contributions: Conceptualization (Vassiliki Siafaka, Periklis Tsekeris, Thomas Hyphantis, and Nafsika Ziavra); methodology (Vassiliki Siafaka and Thomas Hyphantis); data collection (Maria Prodromou and Kleoniki Katinioti); analysis (Dionysios Tafiadis); writing-original data preparation (Vassiliki Siafaka); writing-review and editing (Thomas Hyphantis, Periklis Tsekeris, and Nafsika Ziavra); supervision (Vassiliki Siafaka and Thomas Hyphantis).

Disclosure: All authors read and approved the final manuscript. All authors ensure that there is no author identifying information anywhere in the blinded manuscript, i.e., author details, ethical statements, acknowledgements, author contribution, grant information, funding number, etc. Our research team has the full control of all primary data, and we agree to allow the journal to review our data if requested.

Funding: The study did not receive any specific grant from funding agencies in the public, commercial, or not-for-profit sectors.

Conflict of Interest: The authors report no financial or other conflict of interest relevant to this article, which is the intellectual property of the authors.

Copyright $₫ 2021$ The Author(s). This is an open-access article distributed under the terms of the Creative Commons Attribution 4.0 International License (CC-BY).

\section{REFERENCES}

1. Morris N, Moghaddam N, Tickle A, Biswas S. The relationship between coping style and psychological distress in people with head and neck cancer: A systematic review. Psychooncology 2018;27(3):734-747.

2. Lang $H$, France $E$, Williams B, Humphris G, Wells M. The psychological experience of living with head and neck cancer: A systematic review and meta-synthesis. Psychooncology 2013;22(12):2648-2663.

3. Leventhal H, Diefenbach M, Leventhal EA. Illness cognition: Using common sense to understand treatment adherence and affect cognition interactions. Cognitive therapy research 1992;16(2):143-163.

4. Scharloo M, Baatenburg de Jong RJ, Langeveld TP, van Velzen-Verkaik E, Doorn-op den Akker MM, Kaptein AA. Quality of life and illness perceptions in patients with recently diagnosed head and neck cancer. Head Neck 2005;27(10):857-863.

5. Terrell JE, Ronis DL, Fowler KE, et al. Clinical predictors of quality of life in patients with head and neck cancer. Arch Otolaryngol Head Neck Surg 2004;130(4):401-408.

6. Duffy SA, Terrell JE, Valenstein M, Ronis DL, Copeland LA, Connors M. Effect of smoking, alcohol, and depression on the quality of life of head and neck cancer patients. Gen Hosp Psychiatry 2002;24(3):140-147.

7. Aarstad HJ, Osthus AA, Aarstad HH, Lybak S, Aarstad AKH. General health-related quality of life scores from head and neck squamous cell carcinoma patients obtained throughout the first year following diagnosis predicted up to 10-year overall survival. Eur Arch Otorhinolaryngol 2018;275(1):207-217.

8. Aarstad HJ, Osthus AA, Aarstad HH, Lybak S, Aarstad AKH. EORTC Quality of Life Questionnaire Head and Neck (H\&N)-35 scores from H\&N squamous cell carcinoma patients obtained at diagnosis and at 6,9 and 12 months following diagnosis predict 10-year overall survival. Eur Arch Otorhinolaryngol 2019;276(12):3495-3505.

9. Llewellyn CD, McGurk M, Weinman J. Head and neck cancer: To what extent can psychological factors explain differences between health-related quality of life and individual quality of life? Br J Oral Maxillofac Surg 2006;44(5):351-357.

10. Aarstad AK, Beisland E, Osthus AA, Aarstad HJ. Distress, quality of life, neuroticism and psychological coping are related in head and neck cancer patients during follow-up. Acta Oncol 2011;50(3):390-398.

11. Cavell S, Broadbent E, Donkin L, Gear K, Morton RP. Observations of benefit finding in head and neck cancer patients. Eur Arch Otorhinolaryngol 2016;273(2):479485.

12. Sherman AC, Simonton S, Adams DC, Vural E, Hanna E. Coping with head and neck cancer during different phases of treatment. Head Neck 2000;22(8):787-793.

13. Chen AM, Jennelle RL, Grady V, et al. Prospective study of psychosocial distress 
among patients undergoing radiotherapy for head and neck cancer. Int J Radiat Oncol Biol Phys 2009;73(1):187-193.

14. Zigmond AS, Snaith RP. The hospital anxiety and depression scale. Acta Psychiatr Scand 1983;67(6):361-370.

15. Michopoulos I, Douzenis A, Kalkavoura C, et al. Hospital Anxiety and Depression Scale (HADS): Validation in a Greek general hospital sample. Ann Gen Psychiatry 2008;7:4

16. Moss-Morris R, Weinman J, Petrie K, Horne R, Cameron L, Buick D. The Revised Illness Perception Questionnaire (IPQ-R). Psychology \& Health 2002;17(1):1-16.

17. Giannousi Z, Manaras I, Georgoulias V, Samonis G. Illness perceptions in Greek patients with cancer: A validation of the Revised-Illness Perception Questionnaire. Psychooncology 2010;19(1):85-92.

18. Carver CS, Scheier MF, Weintraub JK. Assessing coping strategies: A theoretically based approach.J Pers Soc Psychol 1989;56(2):267-283.

19. Kapsou M, Panayiotou G, Kokkinos CM, Demetriou AG. Dimensionality of coping: An empirical contribution to the construct validation of the brief-COPE with a Greek-speaking sample. J Health Psychol 2010;15(2):215-229.

20. Aaronson NK, Ahmedzai S, Bergman B, et al. The European Organization for Research and Treatment of Cancer QLQ-C30: A quality-of-life instrument for use in international clinical trials in oncology. J Natl Cancer Inst 1993;85(5):365-376.

21. Bjordal K, Hammerlid E, Ahlner-Elmqvist M, et al. Quality of life in head and neck cancer patients: Validation of the European Organization for Research and Treatment of Cancer Quality of Life Questionnaire-H\&N35. J Clin Oncol 1999;17(3):1008-1019.

22. Jacobi I, van der Molen L, Huiskens H, van Rossum MA, Hilgers FJ. Voice and speech outcomes of chemoradiation for advanced head and neck cancer: A systematic review. Eur Arch Otorhinolaryngol 2010;267(10):1495-1505.

23. Cocks K, King MT, Velikova G, et al. Evidence-based guidelines for interpreting change scores for the European Organisation for the Research and Treatment of Cancer Quality of Life Questionnaire Core 30. Eur J Cancer 2012;48(11):1713-1721.

24. Leong Abdullah MF, Nik Jaafar NR, Zakaria H, et al. Posttraumatic growth, depression and anxiety in head and neck cancer patients: Examining their patterns and correlations in a prospective study. Psychooncology 2015;24(8):894-900.

25. Neilson K, Pollard A, Boonzaier A, et al. A longitudinal study of distress (depression and anxiety) up to 18 months after radiotherapy for head and neck cancer. Psychooncology 2013;22(8):1843-1848.

26. Barber B, Dergousoff J, Nesbitt M, et al. Depression as a predictor of postoperative functional performance status (PFPS) and treatment adherence in head and neck cancer patients: A prospective study. J Otolaryngol Head Neck Surg 2015;44:38.

27. Chen AM, Hsu S, Felix C, Garst J, Yoshizaki T. Effect of psychosocial distress on outcome for head and neck cancer patients undergoing radiation. Laryngoscope 2018;128(3):641-645.

28. Bozec A, Demez P, Gal J, et al. Long-term quality of life and psycho-social outcomes after oropharyngeal cancer surgery and radial forearm free-flap reconstruction: A GETTEC prospective multicentric study. Surg Oncol 2018;27(1):23-30.

29. Ghiggia A, Castelli L, Riva G, et al. Psychological distress and coping in nasopharyngeal cancer: An explorative study in Western Europe. Psychol Health Med 2017;22(4):449-461.

30. Aarstad HJ, Osthus AA, Olofsson J, Aarstad AK. Level of distress predicts subse- quent survival in successfully treated head and neck cancer patients: A prospective cohort study. Acta Otolaryngol 2014;134(2):211-219.

31. Dempster M, McCorry NK, Brennan E, Donnelly M, Murray LJ, Johnston BT. Do changes in illness perceptions predict changes in psychological distress among oesophageal cancer survivors? J Health Psychol 2011;16(3):500-509.

32. Hulbert-Williams NJ, Morrison V, Wilkinson C, Neal RD. Investigating the cognitive precursors of emotional response to cancer stress: Re-testing Lazarus's transactional model. BrJ Health Psychol 2013;18(1):97-121.

33. Dempster M, Howell D, McCorry NK. Illness perceptions and coping in physical health conditions: A meta-analysis. J Psychosom Res 2015;79(6):506-513.

34. Richardson AE, Morton RP, Broadbent E. Coping strategies predict post-traumatic stress in patients with head and neck cancer. Eur Arch Otorhinolaryngol 2016;273(10):3385-3391.

35. Schnoll RA, James C, Malstrom M, et al. Longitudinal predictors of continued tobacco use among patients diagnosed with cancer. Ann Behav Med 2003;25(3):214222.

36. Elani HW, Allison PJ. Coping and psychological distress among head and neck cancer patients. Support Care Cancer 2011;19(11):1735-1741.

37. Howren MB, Christensen AJ, Karnell LH, Funk GF. Psychological factors associated with head and neck cancer treatment and survivorship: Evidence and opportunities for behavioral medicine. J Consult Clin Psychol 2013;81(2):299-317.

38. List MA, Lee Rutherford J, Stracks J, Haraf D, Kies MS, Vokes EE. An exploration of the pretreatment coping strategies of patients with carcinoma of the head and neck. Cancer 2002;95(1):98-104.

39. Paika V, Almyroudi A, Tomenson B, et al. Personality variables are associated with colorectal cancer patients' quality of life independent of psychological distress and disease severity. Psychooncology 2010;19(3):273-282.

40. Aarstad AK, Aarstad HJ, Olofsson J. Quality of life, drinking to cope, alcohol consumption and smoking in successfully treated HNSCC patients. Acta Otolaryngol 2007;127(10):1091-1098.

41. Llewellyn CD, McGurk M, Weinman J. Illness and treatment beliefs in head and neck cancer: Is Leventhal's common sense model a useful framework for determining changes in outcomes over time? J Psychosom Res 2007;63(1):17-26.

42. Mazzotti E, Sebastiani C, Marchetti P. Patient perception of disease control and psychological distress. Cancer Manag Res 2012;4:335-340.

43. Richardson EM, Schuz N, Sanderson K, Scott JL, Schuz B. Illness representations, coping, and illness outcomes in people with cancer: A systematic review and meta-analysis. Psychooncology 2017;26(6):724-737.

44. Hagger MS, Koch S, Chatzisarantis NLD, Orbell $S$. The common sense model of self-regulation: Meta-analysis and test of a process model. Psychol Bull 2017;143(11):1117-1154.

45. Richardson AE, Tennant G, Morton RP, Broadbent E. A self-regulatory intervention for patients with head and neck cancer: Pilot randomized trial. Ann Behav Med 2017;51(5):629-641.

46. Dunne S, Mooney O, Coffey L, et al. Psychological variables associated with quality of life following primary treatment for head and neck cancer: A systematic review of the literature from 2004 to 2015. Psychooncology 2017;26(2):149-160.

47. Luckett T, Britton B, Clover K, Rankin NM. Evidence for interventions to improve psychological outcomes in people with head and neck cancer: A systematic review of the literature. Support Care Cancer 2011;19(7):871-881. 